\title{
Effect of treadmill exercise on pain-related Wnt/ $\beta$-catenin signaling pathway in dorsal root ganglion neurons at the early phase regeneration of the injured sciatic nerve
}

\author{
Yeong-Hyun Cho, Ji-Eun Kim, Tae-Beom Seo* \\ Department of Kinesiology, College of Natural Science, Jeju National University, Jeju, Korea
}

The purpose of this study was to determine whether treadmill walking exercise can improve mechanical allodynia through regulation of Wnt/ $\beta$-catenin signaling in dorsal root ganglion (DRG) neurons at the early stage of regeneration after sciatic nerve injury (SNI). The experimental rats were divided into seven groups: the normal control, sedentary groups for 3-, 7-, and 14-day post crush (dpc), and exercise group for 3 , 7 , and $14 \mathrm{dpc}$. The rats in exercise groups performed treadmill walking exercise at a speed of $8 \mathrm{~m} / \mathrm{min}$ for 20 min once a day according to experiment duration. For evaluating neuropathic pain-like behavior after SNI, the mechanical allodynia was examined by von Frey apparatus. And the expression levels of pain-related protein were identified in the cytoplasm or nucleus of DRG neurons using Western blot techniques. Mechanical allodynia was significantly ameliorated in the exercise group at 7 and $14 \mathrm{dpc}$. Treadmill exercise further decreased Wnt3a expression at 3, 7, and $14 \mathrm{dpc}$ compared to in the sedentary group. Also, phosphorylated-low-density lipoprotein receptor 6 was decreased in exercise groups at 3 and $14 \mathrm{dpc}$. Beta-catenin was significantly decreased in exercise groups at 3 and $14 \mathrm{dpc}$ compared to sedentary groups as well as treadmill exercise decreased translocation of $\beta$-catenin towards the nucleus of DRG neurons at $14 \mathrm{dpc}$. Our findings indicate that treadmill walking exercise may be an important regulator of neuropathic pain after peripheral nerve injury through delayed Wnt/ $\beta$-catenin signaling pathway in DRG neurons.

Keywords: Sciatic nerve, Neuropathic pain, Treadmill exercise, Wnt3a, $\beta$-catenin

\section{INTRODUCTION}

Neuropathic pain is often pronounced as a shooting or burning pain, which is caused by damage affecting the central and peripheral nervous systems (Kumar et al., 2018). In general, peripheral neuropathic pain is subdivided into dysesthesia and allodynia according to symptoms and signs. Dysesthesia involves a disagreeable sensation such as a painful burning, prickling or aching feeling, and allodynia is defined as an unpleasant sensation that occurs with light touch. Sciatic nerve injury (SNI) is more closely related to allodynia than to dysesthesia (Mukai et al., 2019). Patients with neuropathic pain after SNI have suffered from poor quality of life due to anxiety, depression and severe pain (Bernetti et al., 2021). Therefore, many researchers have tried to find out mechanisms of neuropathic pain for several decades (Finnerup et al., 2021), but the mechanism is not yet clear.

According to previous studies on peripheral pain mechanisms reported until recently, it has been reported that Wnt signaling pathway is associated with neuropathic pain, classified into the canonical Wnt/ $\beta$-catenin pathway and the noncanonical $\beta$-cateninindependent pathway (Itokazu et al., 2014). The canonical Wnt pathway results in an accumulation of $\beta$-catenin in the cytoplasm through binding several transmembrane receptors, including the frizzled receptor (FZ), low-density lipoprotein receptor 6 (LRP6) and low-density lipoprotein receptor 5 (LRP5), and stabilized $\beta$-catenin is translocated into the nucleus to activate transcription factors (Zhao and Yang, 2018), while the noncanonical pathway does not involve $\beta$-catenin as well as it not binding with LRP5

\footnotetext{
${ }^{*}$ Corresponding author: Tae-Beom Seo (i) https://orcid.org/0000-0001-9213-2251 Department of Kinesiology, College of Natural Science, Jeju National University, 102 Jejudaehak-ro, Jeju 63243, Korea

Email: seotb@jejunu.ac.kr

Received: March 3, 2021 / Accepted: March 17, 2021
}

This is an Open Access article distributed under the terms of the Creative Commons Attribution Non-Commercial License (https://creativecommons.org/licenses/by-nc/4.0/) which permits unrestricted non-commercial use, distribution, and reproduction in any medium, provided the original work is properly cited. 
and LRP6 (Komiya and Habas, 2018).

In studies on central nerve degeneration and SNI, the canonical Wnt pathway has been known as an indicator of pain that increases inflammatory cytokines such as the nuclear factor kappa light chain enhancer of activated B cell (NF-kB), tumor necrosis factor- $\alpha$ (TNF- $\alpha$ ), and interleukin- 6 in the dorsal root ganglion (DRG) and the dorsal horn of the spinal cord (L'episcopo et al., 2011; Marchetti and Pluchino, 2013; Xu et al., 2018; Zhang et al., 2010). Dworkin et al. (2007) suggested that inhibition of Wnt protein expression through drug treatment and surgery resulted in a decrease in threshold of mechanical allodynia in about $30 \%$ of patients with neuropathic pain. However, these treatment methods have a large economic burden compared to their effectiveness (Bussa et al., 2021).

Regular aerobic exercise including walking, running and swimming has been used as one of the therapeutic strategies for motor and/or sensory recovery of damaged nerves in the field of the neuropathology and neurology (Sarikcioglu et al., 2009). Looking at some previous studies that confirmed the effect of exercise on neuropathic pain, it was reported that both forced and voluntary exercise not only increased the expression level of the glial cell-derived neurotrophic factor (GDNF) in the injured nerves but it also decreased the incidence of mechanical allodynia by $7 \%$ after spinal cord injury (Detloff et al., 2014) and peripheral nerve injury (LópezÁlvarez et al., 2015). Furthermore, Shen et al. (2013) reported that swimming exercise attenuated inflammation and peripheral neuropathic pain.

With these results found in previous studies, continuous physical activity is a positive effector closely related to neuropathic pain and mechanical allodynia after nervous system injury. However, research on exercise-regulated $\mathrm{Wnt} / \beta$-catenin signaling at early phase regeneration of the injured sciatic nerve is still insufficient. Therefore, the purpose of this study focuses on investigating the effect of treadmill training on the pain-related Wnt/ $\beta$-catenin signaling pathway in lumbar 4 to 5 DRG neurons at the early stage of regeneration after $\mathrm{SNI}$.

\section{MATERIALS AND METHODS}

\section{Experimental animals}

Male Sprague-Dawley rats (150-160 g, 6 weeks old) were used in this experiment. They were randomly divided into seven groups: the normal control, sedentary groups for 3-, 7-, 14-day post crush (dpc), and exercise group for 3, 7, $14 \mathrm{dpc}(\mathrm{n}=10$ in each group). Animals were maintained at a constant room temperature of $22^{\circ} \mathrm{C}$ and $60 \%$ of humidity under 12/12-hr light-dark cycle. They were accepted to eat commercial rat chow (Samyang Co., Seoul, Korea) and water ad libitum. This experiment obtained approval by the Ethics Committee of Jeju National University (2018-0028).

\section{Sciatic nerve injury}

The rats in the injury group were anesthetized using an animal inhalation narcosis control (Jeungdo Bio \& Plant, Seoul, Korea). First, the rats were placed into the chamber with a $2 \%-2.5 \%$ concentration of isoflurane for anesthesia and then $1.5 \%-1.8 \%$ concentration for maintenance during SNI. The left sciatic nerve was crushed with a pair of forceps held tightly for $1 \mathrm{~min}$ and $30 \mathrm{sec}$ at intervals (Seo et al., 2006). After surgery, anesthetized animals were then placed on a heating pad maintained at $37^{\circ} \mathrm{C}$, and then they were put in their cages for resting. All rats were sacrificed 3, 7, and 14 days later.

\section{Treadmill exercise}

All rats used in the experiment were adapted to treadmill walking exercise for a week before the study began. Excluding the control group, sciatic nerve surgery was performed in the exercise and sedentary groups. After resting for 2 days, all rats in the exercise group performed a low-intensity walking exercise on the treadmill device (Jeungdo Bio \& Plant) on $8 \mathrm{~m} / \mathrm{min}$ for $20 \mathrm{~min}$ with no inclination during experiment duration $(3,7$, and $14 \mathrm{dpc})$.

\section{Western blot analysis}

Lumbar 4 to 5 DRG neurons play a role in transmitting the sensory signal into the central nervous system. The dissected DRG were rinsed with phosphate-buffered saline, and lysed in Triton lysis buffer. The nucleus and cytoplasm were separated by nuclear extraction buffer and cytosol extraction buffer. Denatured proteins were separated on sodium dodecyl sulphate-polyacrylamide gel and then transferred onto polyvinylidene difluoride membrane on ice at $200 \mathrm{~mA}$ for $2 \mathrm{hr}$. The membranes were blocked with 5\% skim milk, $0.1 \%$ Tween 20 in tris buffered saline for $30 \mathrm{~min}$ at room temperature. Then, the membranes were incubated overnight with primary antibodies at $4{ }^{\circ} \mathrm{C}$. Protein $(20 \mu \mathrm{g})$ was used for Western blot analysis using anti-mouse Wnt3a (1:1,000, Cell Signaling Biotechnology, Danvers, MA, USA), anti-rabbit phosphorylated LRP6 (1:1,000, Cell Signaling Biotechnology), anti-mouse LRP6 (1:1,000, Cell Signaling Biotechnology), anti-mouse $\beta$-catenin (1:1,000, Santa Cruz Biotechnology, Santa Cruz, CA, USA), antimouse $\beta$-actin (1:2000, Santa Cruz Biotechnology) antibodies. For the secondary antibody, Horseradish peroxidase-conjugated anti- 
mouse or anti-rabbit IgG antibodies (1:1,000, GenéTex Inc., Irvine, CA, USA) were used. The blotting proteins were detected by using Westar ECL substrates (Cyanagen, Bologna, Italy). Detected band intensity was analyzed using Chemidoc (Bio-Rad, Hercules, CA, USA).

\section{Paw withdrawal test}

For assessment of mechanical allodynia, rats were placed on a clear individual box with holes and the animals were allowed to adapt for $30 \mathrm{~min}$ before the test. The von Frey filament (BIOEVF4, Bioseb, Vitrolles, France), plastic hairs of calibrated diameters, was applied to the plantar surface of the left hind paw in a series of ascending forces (Yalcin et al., 2014). All rats were always tested for 3 times and the time and intensity of hind paw withdrawal was measured. The filaments that were the most often used are $0.16,0.4,0.6,1,1.4,2,4,6,8$, and $10 \mathrm{~g}$. The von Frey test was performed before the injury ( 0 day), 3,7 , and $14 \mathrm{dpc}$.

\section{Statistical analysis}

All the data is presented as a mean \pm standard error. Statistical analysis was performed using one-way analysis of variance followed by Duncan post boc test. The significance level was set at $P<0.05$. All data analysis and graphs were performed by using Prism 6 (GraphPad, La Jolla, CA, USA).

\section{RESULTS}

\section{Treadmill exercise improved mechanical allodynia after SNI}

To confirm the effect of treadmill exercise on peripheral painlike behavior after SNI, we analyzed the time-dependent effect on latency for hind paw withdrawal using the von Frey test device.
As shown in Fig. 1, exercise groups at 7 and 14 dpc significantly ameliorated mechanical allodynia compared to the sedentary groups, but there was no significant difference between exercise and sedentary group at $3 \mathrm{dpc}$.

\section{Treadmill exercise decreased Wnt3a and p-LRP6 levels in the DRG neurons after SNI}

Wnt3a and LRP6 have been known as a pain marker of the injured nervous system. To investigate the change of pain-related proteins after SNI, we analyzed the time-dependent effect of treadmill exercise on Wnt3a and LRP6 in DRG neurons after SNI. As shown in Fig. 2, Wnt3a was significantly decreased in treadmill exercise groups at 3, 7, and $14 \mathrm{dpc}$ compared to sedentary groups. LRP6 protein showed no significant difference between sedentary and exercise groups at all time points, while phosphorylated p-LRP6 in exercise groups turned out to be significantly lower than those in sedentary groups at 3 and $14 \mathrm{dpc}$.

\section{Treadmill exercise regulates translocation of $\beta$-catenin to nucleus of DRG neurons after SNI}

To examine translocation of $\beta$-catenin from the cytoplasm to the nucleus of DRG neurons after SNI, we analyzed alterations of $\beta$-catenin in lumbar 4 to 5 DRG neurons and then confirmed a quantitative measure of transported $\beta$-catenin in cytoplasm and nucleus of DRG neurons. As shown in Fig. 3, $\beta$-catenin was significantly decreased in exercise groups at 3 and $14 \mathrm{dpc}$ compared to sedentary groups. In the cytoplasm of DRG neurons, $\beta$-catenin did not indicate a significant difference between sedentary and exercise groups at all time points, while translocation of $\beta$-catenin to the nucleus of neurons further decreased in exercise groups $14 \mathrm{dpc}$ compared to those in sedentary groups.
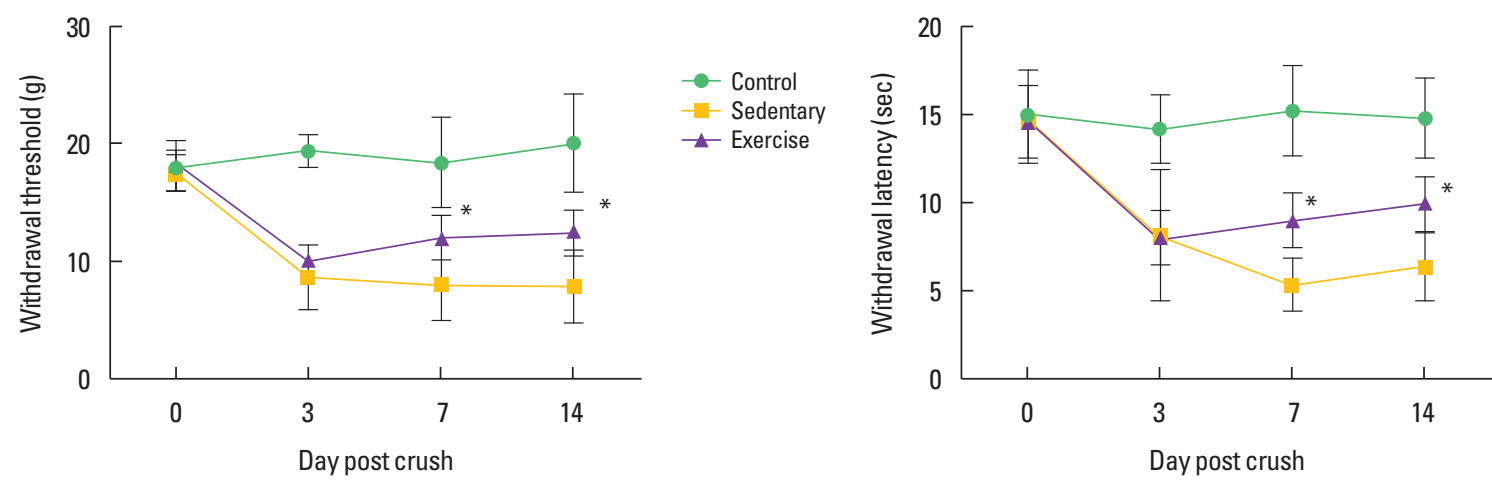

Fig. 1. Treadmill exercise improved mechanical allodynia after sciatic nerve injury. Hind paw withdrawal test was measured in grams (g) and times (sec) using von Frey device at baseline (day 0) and on days 3, 7, and 14 post injury. Treadmill exercise improved the mean withdrawal threshold of the ipsilateral hind paw on day 7 and 14 after injury. The left panel shows the finding on grams (g) and the right on the time (sec). ${ }^{*} P<0.05$ vs. the sedentary group. 


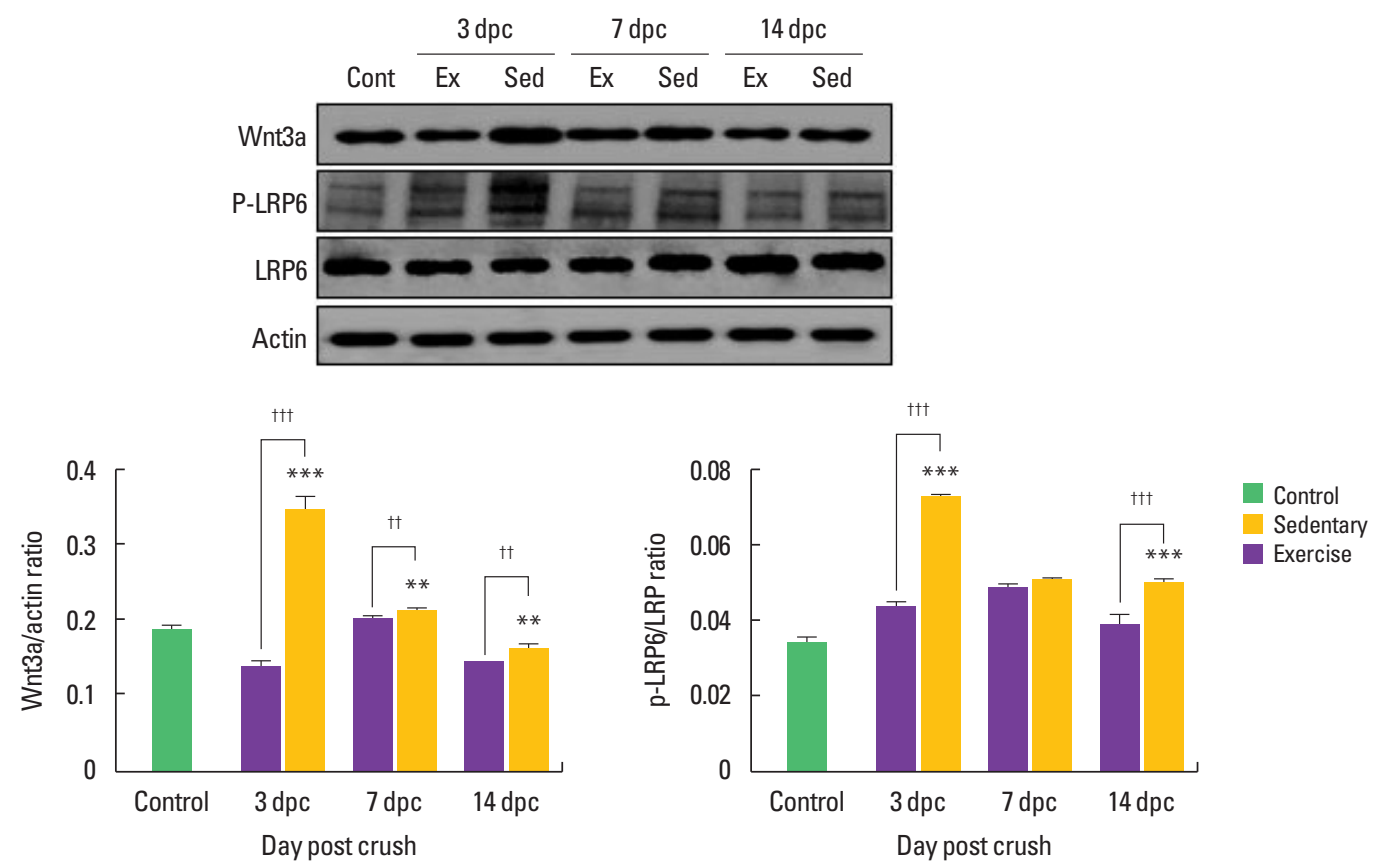

Fig. 2. Continuous exercise downregulated activation of Wnt3a-low-density lipoprotein receptor 6 (LRP6) signaling pathway in dorsal root ganglion neurons at early phase of axonal regeneration after sciatic nerve injury. Wnt3a protein was significantly downregulated in exercise group at all time points of regeneration. In particular, it was sharply reduced at $3 \mathrm{dpc}$. Treadmill walking significantly decreased $p$-LRP6 expression levels at 3 and $14 \mathrm{dpc}$. ${ }^{* *} P<0.01,{ }^{* * *} P<0.001$ vs. the control group. ${ }^{\dagger} P<0.01,{ }^{t \dagger t} P<0.001$ vs. the sedentary group. dpc, days post crush; Cont, control group; Ex, treadmill exercise group; Sed, sedentary group.
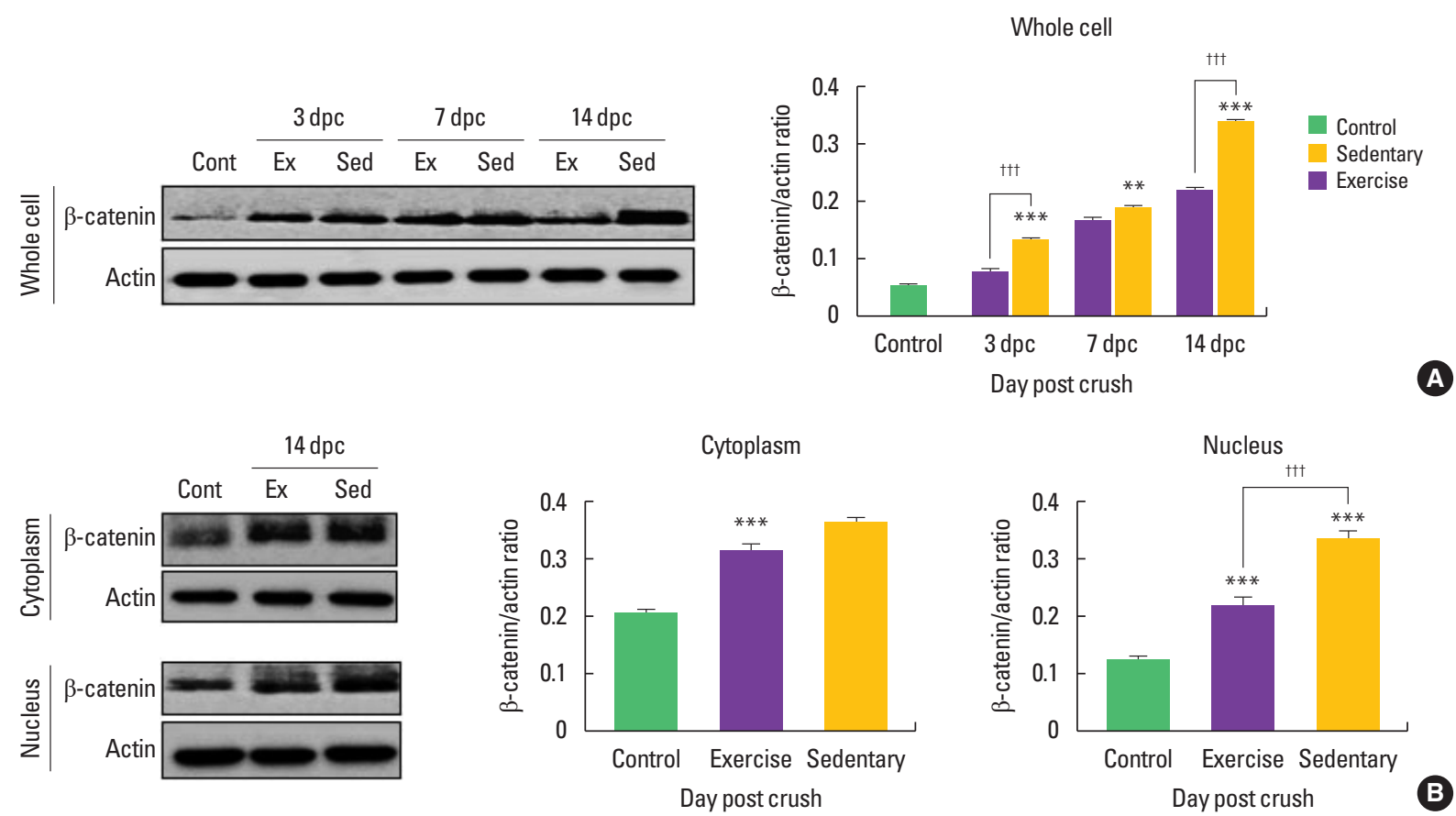

Fig. 3. Treadmill walking exercise accelerated translocation of $\beta$-catenin towards the nucleus of dorsal root ganglion neurons after sciatic nerve injury. (A) In whole cell lysate, $\beta$-catenin was decreased in exercise group compared to those in the sedentary group at 3 and $14 \mathrm{dpc}$. In particular, $\beta$-catenin showed the greatest tendency to decrease in the 14-day exercise group. (B) We reevaluated $\beta$-catenin expression in the cytoplasm or nucleus of dorsal root ganglion neurons at 14 days after sciatic nerve injury. Treadmill exercise results in rapid translocation of $\beta$-catenin towards the nucleus at $14 \mathrm{dpc} .{ }^{* *} P<0.01$. ${ }^{* * *} P<0.001 \mathrm{vs}$. the control group. ${ }^{t+\dagger} P<0.001$ vs. the sedentary group. dpc, days post crush; Cont, control group; Ex, treadmill exercise group; Sed, sedentary group. 


\section{DISCUSSION}

Although many previous studies reported that aerobic and anaerobic exercises are effective in reducing neuropathic pain after SNI, these findings do not suggest a specific mechanism of exercise for mechanical allodynia in the early stage of axonal nerve regeneration (Kami et al., 2017). Therefore, this study investigated pain-related biochemical and behavioral changes through exercise in the early stage of regeneration.

Neuropathic pain in nervous system disorders is a pain condition caused by abnormal sensations called dysesthesia and allodynia. Peripheral nerve damage including SNI usually produces allodynia rather than dysesthesia (Bourquin et al., 2006). Yalcin et al. (2014) represented that paw withdrawal threshold in the von Frey test would be the most reliable method of mechanical allodynia induced after SNI. Thus, we applied mechanical allodynia assessment using the von Frey test device, and confirmed that treadmill exercise dramatically decreased the symptoms of mechanical allodynia at all time points compared to sedentary groups. In previous studies on neuropathic pain-like behavior after peripheral nerve injury, Farzad et al. (2018) suggested that regular exercise for 4 weeks resulted in a beneficial effect on neuropathic pain symptoms after peripheral nerve injury through activation of irisin and glutamic acid decarboxylase-65 protein. But Whitehead et al. (2017) reported conflicting results that acute wheel-running activity did not alter low-intensity mechanical allodynia in the sciatic chronic constriction injury model. The different results between the previous studies and this study are thought to be due to differences in the SNI method, regenerative phase, duration of exercise application, exercise type and exercise intensity.

For decade, many researches on the role of Wnt signaling into the nervous system have made extraordinary advancement with respect to our understanding of embryonic brain development, mammalian neurogenesis, and tumorigenesis (Girardi and Le Grand 2018; Hu et al., 2019). The Wnt protein has been well known as an indicator that underlies the pathogenesis of neuropathic pain in rodents. Central or peripheral nervous injury leads to a rapid-onset induction of $\mathrm{Wnt}$ in the primary sensory neurons (Zhang et al., 2013). Wnt co-receptor LRP6 also is dramatically increased in association with activated Wnt signaling (Simonetti et al., 2014). In our study, the prototype canonical Wnt3a and LRP6 in the exercise group showed a tendency to decrease continuously from 3 days until 14 days after SNI. Inceoglu et al. (2015) confirmed that Wnt inhibitors improved mechanical allodynia and thermal hyperalgesia in neuropathic pain models as well as swimming exercise decreased on expression of Wnt3a-LRP proteins in Wallerian degeneration condition. These previous studies and present findings imply that regular exercise can be a positive effector on the blocking of neuropathic pain-related protein expression after $\mathrm{SNI}$.

In addition to induction of Wnt and LRP6, $\beta$-catenin also is a multifunctional protein that activates Wnt signals in rat model with neuropathic pain (Peng et al., 2019). We established that treadmill exercise downregulated translocation of $\beta$-catenin to nucleus of DRG neurons after SNI. During neuropathic pain stage, $\beta$-catenin is mainly activated in neurons by binding of $\mathrm{Wnt}$ and $\mathrm{FZ}$ receptors, and it is translocated from cytoplasm to nucleus for activating T-cell factor/lymphoid enhancer factor series transcription factors (Wisniewska et al., 2010). In other words, nuclear translocation of $\beta$-catenin in DRG neurons can aggravate mechanical allodynia induced by SNI. Thus, present study that treadmill exercise suppressed nuclear accumulation of $\beta$-catenin in DRG cells to regulate mechanical allodynia was consistent with some previous studies showing that the $\mathrm{Wnt} / \beta$-catenin should be blocked to improve neurotrophic pain (Zhang et al., 2013), suggesting that exercise may be a potential regulator for attenuating neuropathic pain.

Given these findings reported in the present study, regular treadmill exercise would be effective in controlling neuropathic pain including mechanical allodynia. In conclusion, the present finding provided critical evidence that physical exercise might regulate neuropathic pain after peripheral nerve injury through delayed Wnt/ $\beta$-catenin signaling pathway in DRG neurons.

\section{CONFLICT OF INTEREST}

No potential conflict of interest relevant to this article was reported.

\section{ACKNOWLEDGMENTS}

This work was supported by the Ministry of Education of the Republic of Korea and the National Research Foundation of Korea (NRF- 2018S1A5A8028577).

\section{REFERENCES}

Bernetti A, Agostini F, de Sire A, Mangone M, Tognolo L, Di Cesare A, Paoloni M. Neuropathic pain and rehabilitation: a systematic review of international guidelines. Diagnostics (Basel) 2021;11:74. 
Bourquin, AF, Süveges M, Pertin M, Gilliard N, Sardy S, Davison AC, Decosterd I. Assessment and analysis of mechanical allodynia-like behavior induced by spared nerve injury (SNI) in the mouse. Pain 2006; 122:14.e1-e14.

Bussa M, Mascaro A, Sbacchi E, Dourandish M, Rinaldi S. Understanding peripheral neuropathic pain in primary care: diagnosis and management. Eur Rev Med Pharmacol Sci 2021;25:1990-1996.

Detloff MR, Smith EJ, Quiros Molina D, Ganzer PD, Houlé JD. Acute exercise prevents the development of neuropathic pain and the sprouting of non-peptidergic (GDNF-and artemin-responsive) c-fibers after spinal cord injury. Exp Neurol 2014;255:38-48.

Dworkin RH, Jensen MP, Gammaitoni AR, Olaleye DO, Galer BS. Symptom profiles differ in patients with neuropathic versus non-neuropathic pain. J Pain 2007;8:118-126.

Farzad B, Rajabi H, Gharakhanlou R, Allison DJ, Hayat P, Jameie SB. Swimming training attenuates allodynia and hyperalgesia induced by peripheral nerve injury in an adult male rat neuropathic model: effects on irisin and GAD65. Pain Med 2018;19:2236-2245.

Finnerup NB, Kuner R, Jensen TS. Neuropathic pain: from mechanisms to treatment. Physiol Rev 2021;101:259-301.

Girardi F, Le Grand F. Wnt signaling in skeletal muscle development and regeneration. Prog Mol Biol Transl Science 2018;153:157-179.

Hu S, Yang L, Wu C, Liu TY. Regulation of Wnt signaling by physical exercise in the cell biological processes of the locomotor system. Physiol Int 2019;106:1-20.

Inceoglu B, Bettaieb A, Trindade da Silva CA, Lee KS, Haj FG, Hammock $\mathrm{BD}$. Endoplasmic reticulum stress in the peripheral nervous system is a significant driver of neuropathic pain. Proc Natl Acad Sci U S A 2015; 112:9082-9087.

Itokazu T, Hayano Y, Takahashi R, Yamashita T. Involvement of Wnt/ $\beta$-catenin signaling in the development of neuropathic pain. Neurosci Res 2014;79:34-40.

Kami K, Tajima F, Senba E. Exercise-induced hypoalgesia: potential mechanisms in animal models of neuropathic pain. Anat Sci Int 2017;92: 79-90.

Komiya Y, Habas R. Wnt signal transduction pathways. Organogenesis 2018;4:68-75.

Kumar A, Kaur H, Singh A. Neuropathic pain models caused by damage to central or peripheral nervous system. Pharmacol Rep 2018;70:206216.

L'episcopo F, Serapide MF, Tirolo C, Testa N, Caniglia S, Morale MC, Pluchino S, Marchetti B. A Wnt1 regulated Frizzled-1/ $\beta$-Catenin signaling pathway as a candidate regulatory circuit controlling mesencephalic dopaminergic neuron-astrocyte crosstalk: Therapeutical relevance for neuron survival and neuroprotection. Mol Neurodegener
2011;6:49.

López-Álvarez VM, Modol L, Navarro X, Cobianchi S. Early increasingintensity treadmill exercise reduces neuropathic pain by preventing nociceptor collateral sprouting and disruption of chloride cotransporters homeostasis after peripheral nerve injury. Pain 2015;156:1812-1825.

Marchetti B, Pluchino S. Wnt your brain be inflamed? Yes, it Wnt! Trends Mol Med 2013;19:144-156.

Mukai M, Uchida K, Hirosawa N, Murakami K, Kuniyoshi K, Inoue G, Miyagi M, Sekiguchi H, Shiga Y, Inage K, Orita S, Suzuki T, Matsuura Y, Takaso M, Ohtori S. Wrapping with basic fibroblast growth factorimpregnated collagen sheet reduces rat sciatic nerve allodynia. J Orthop Res 2019;37:2258-2263.

Peng Z, Zha L, Yang M, Li Y, Guo X, Feng Z. Effects of ghrelin on pGSK$3 \beta$ and $\beta$-catenin expression when protects against neuropathic pain behavior in rats challenged with chronic constriction injury. Sci Rep 2019;9:14664.

Sarikcioglu L, Demirel BM, Utuk A. Walking track analysis: an assessment method for functional recovery after sciatic nerve injury in the rat. Folia Morphol (Warsz) 2009;68:1-7.

Seo TB, Han IS, Yoon JH, Hong KE, Yoon SJ, Namgung UK. Involvement of $\mathrm{Cdc} 2$ in axonal regeneration enhanced by exercise training in rats. Med Sci Sports Exerc 2006;38:1267-1276.

Shen J, Fox LE, Cheng J. Swim therapy reduces mechanical allodynia and thermal hyperalgesia induced by chronic constriction nerve injury in rats. Pain Med 2013;14:516-525.

Simonetti M, Agarwal N, Stösser S, Bali KK, Karaulanov E, Kamble R, Heppenstall P. Wnt-Fzd signaling sensitizes peripheral sensory neurons via distinct noncanonical pathways. Neuron 2014;83:104-121.

Whitehead RA, Lam NL, Sun MS, Sanchez JJ, Noor S, Vanderwall AG, Milligan ED. Chronic sciatic neuropathy in rat reduces voluntary wheel running activity with concurrent chronic mechanical allodynia. Anesth Analg 2017;124:346-355.

Wisniewska MB, Misztal K, Michowski W, Szczot M, Purta E, Lesniak W, Mozrzymas JW. LEF1/ $\beta$-catenin complex regulates transcription of the Cav3.1 calcium channel gene (Cacna1g) in thalamic neurons of the adult brain. J Neursci 2010;30:4957-4969.

Xu L, Liu Y, Sun Y, Li H, Mi W, Jiang Y. Analgesic effects of TLR4/NF- $k B$ signaling pathway inhibition on chronic neuropathic pain in rats following chronic constriction injury of the sciatic nerve. Biomed Pharmacother 2018;107:526-533.

Yalcin E, Unlu E, Akyuz M, Karaahmet OZ. Ultrasound diagnosis of ulnar neuropathy: comparison of symptomatic and asymptomatic nerve thickness. J Hand Surg Eur Vol 2014;39:167-171.

Zhang L, Fu ZJ, Sun T, Zhao XL, Song WG, Jia MR, Wei GF. Expression of NF- $\kappa B$ and TNF- $\alpha$ in spinal dorsal horn in a rat model of neuropathic 
pain. Zhonghua Yi Xue Za Zhi 2010;90:1067-1071.

Zhang YK, Huang ZJ, Liu S, Liu YP, Song AA, Song XJ. WNT signaling underlies the pathogenesis of neuropathic pain in rodents. J Clin In- vestig 2013;123:2268-2286.

Zhao Y, Yang Z. Effect of Wnt signaling pathway on pathogenesis and intervention of neuropathic pain. Exp Ther Med 2018;16:3082-3088. 